\title{
Impact of MRAI Timer on BGP Updates and Convergence Time
}

\author{
R. N. Devikar ${ }^{*}$, D. V. Patil ${ }^{* *}$, and V. Chandraprakash ${ }^{* * *}$ \\ * Research Scholar, Department of CSE, Koneru Lakshmaiah Education Foundation, Vaddeswaram, Guntur, AP, India \\ ${ }^{* *}$ Department of Computer Engineering, MET IOE, Bhujbal Knowledge City, Pune University \\ ${ }^{* * *}$ Professor, Department of CSE, Koneru Lakshmaiah Education Foundation, Vaddeswaram, Guntur, AP., India
}

\section{Article Info}

\section{Article history:}

Received October 16, 2017

Revised August 24, 2018

Accepted

\section{Keyword:}

Convergence Time

MRAI

FMRAI

Inter-domain routing

\begin{abstract}
BGP is a vital routing protocol for the communication amongst autonomous systems in the internet and has been broadly applied in all categories of large scale network. The interdomain routing protocol (BGP) shows slow convergence, which effects on many internet applications due to its high convergence delay. The network operators broadly use different MRAI timers in BGP routers to deal with the issue of growing convergence time of the network. The variation in MRAI timer and its impact on network convergence and update messages has been broadly studied over the years. The increasing size of autonomous systems leads to rise in number of MRAI timers. Hence, the optimum use of MRAI timers can decrease the problem of slow convergence and necessity of huge number of MRAI timers. The proposed system uses the ckle minimum route advertisement interval timer (FMRAI) for fast update of routing table, which leads to reduce the convergence time of a network. In comparison with static MRAI timer of 30s the FMRAI timer leads to better result in terms of convergence time and number of update messages.
\end{abstract}

Copyright (C) 2018 Institute of Advanced Engineering and Science. All rights reserved.

\author{
Corresponding Author: \\ Rohit Nilkanth Devikar \\ Research Scholar \\ Departement of Computer Science and Engineering \\ Koneru Lakshmaiah Education Foundation, Vaddeswaram, Guntur, AP, India \\ +919028339491 \\ rohit.devikar89@gmail.com
}

\section{INTRODUCTION}

The Border Gateway Protocol (BGP) is an inter-domain routing protocol. The BGP is use in the autonomous system (AS). Each and every AS is identified by autonomous system number (ASN). The router which is used to connect two different AS is called BGP speaker node. The primary functionality of a BGP speaker node is to exchange network reachability information with other BGP speaker node of neighbour autonomous system. This network reachability information contains information on the list of Autonomous Systems (AS) that traverses the reachability information [1], [2]. This information is sufficient to construct a graph of AS connectivity from which routing loops may be pruned and some policy decisions at the AS level may be enforced.

BGP use a bulky number of features to spread the information through the AS path. Such AS-path, records this pathway through all the autonomous systems, hence it is to be regarded as path vector protocol. Currently, Internet is facing many problems because of network instability. Routing instability will causes increasing convergence time, network message delay and may even cause the entire regional network interruption. As the network is scaling rapidly, the topology become more and more complex. Due to this fast routing convergence of BGP is a serious issue now days. However, the BGP protocol is sensitive to definite Internet topological characteristics; it still remains totally insensitive to the deviation of other characteristics. Whenever there is a topology change, which may happen due to policy changes, broken links or connections, or the arrival of a new node, the convergence process is activated, and while a stable state is not reached, packet losses and inconsistencies might take place. During this period, the speedy fluctuation in the network reachability characterizes the routing instability [2]. 
The problem of convergence can be roughly divided into two categories:

1. Routing convergence due to the strategy conflict between ASs, such problem generally does not involve AS internal structure;

2. Another is routing oscillation because of some faultiness of BGP system, which is related to the AS internal structure.

When such fluctuations are happened in the networks, BGP may take long duration to converge. The high convergence delay offered on the Internet acquires in service unavailability with packet loss and poor quality for applications. With the growth of many real-time applications on the Internet such as network banking, Skype, and video conferences, increases the traffic demand. The slow convergence of BGP, for example, seriously impacts on VOIP service, accounting for almost $90 \%$ of the dropped calls. In the last few years, a number of efforts have been made to improve inter-domain routing convergence, proposing BGP modifications, changes on inter-domain architecture or new routing protocols. One problem BGP has encountered, due to the decentralized nature, is its slow converging speed. According to studies in [1], after a topology change, it takes an average of 3 minutes for the Internet to update all the routing tables, with a worst case of 15 minutes. Such a delay may lead to extreme packet losses, and is thus not acceptable to many delay-sensitive applications. Therefore, speeding up BGP convergence is a critically important and yet challenging problem. Our research is based on improving the convergence time of a network by introducing fickle Mean route advertisement interval timer (FMRAI). Literature survey shows that many trials have been done to improve convergence by reducing minimum route advertisement interval (MRAI) timer. But they failed in dynamically changing topology.

In the Internet, network failures happen commonly and the existing routing protocols can take several duration to converge after a failure [3]. During convergence times, several packets may be loss or may already be en-route to their destinations. These in-flight packets can encounter routing loops, delays and packet losses. However, due to this the main question raised how many packets are lost or not reach to the given destination during convergence periods? In this paper, we study the impact of MRAI timer on convergence time and number of updates. The MRAI timer is directly proportional to the convergence time and inversely proportional to the number of updates. Increase in MRAI increases the convergence time, but decreases the number of updates. In literature survey we study and examined many routing protocols those are work to reduce the MRAI timer. Two main aspects in designing the routing protocol, keeping alternative path transmission information at every router and rapidly spreads new reachability information, appear to have the more impact on the packet delivery behaviour during convergence.

Network instability is one of the major problem in currently changing network topology. Instability in the network results in loss of packets, which in turn increases the latency and convergence time. Following section describes the research work done by different researchers to improve the convergence time [4] and instability of the network.

Griffin et al. [5] explained a study on the stable paths and conclude that it is always possible to converge BGP network by using three methodologies.

1. The first is by using operational guidelines, which is a gathering of rules that all ASes must follow to confirm policy safety and correctness.

2. The second approach is based on static analysis, making programs analyse routing policies and detect if any policy conflict or other inconsistency exists, which may cause protocol divergence.

3. The third approach is a dynamic detection, which avoids and suppresses routing fluctuation as soon as it is detected.

RFD uses this third approach, but the authors know its issues and do not recommend its use. To identify potential oscillation on network, Cittadini et al. [6] introduced a heuristic algorithm that performs static detection in an AS. Fabrikant et al. [7] observed that although the default value of MRAI timer is 30 seconds, router vendors are eliminating or lowering the timer, expecting that with a small value of MRAI, the convergence time will be reduced. The authors illustrate that by decrementing MRAI timer does not improve network convergence time, and the behaviour of a network in convergence process might actually get even poorer. So, the deployment of MRAI variations may be done carefully. Elmokash et al. [8] observed that the churns of BGP updates are caused by an interaction of three factors: 1. The routing protocol, which has the BGP technique such as MRAI, RFD and routing policies, etc.

2. Events like BGP updates, link failures, traffic engineering operations etc.

3. The features of the Internet topology.

The authors focused on the impact of the third factor, i.e. topology, on the existence of BGP churn. They created a topology generator to examine churns at different locations of the generated topologies, and analysed the number of updates with increase in size of each topology. Schrieck et al. [9] detected some factors that may cause BGP churn issues. First, certain inter-domain links are frequently failed and unstable, transmitting this information to neighboring ASes through withdrawals. Second, when routes are unavailable BGP suffers from path exploration. In 
addition to this factor, MRAI and RFD may also be foundation to BGP churn and further delay convergence. Elmokash et al. [10] experiment the study on BGP churn evolution in four networks at the Internet backbone during a period of seven years and eight months. By taking an experimental data analysis author suggested that duplicate announcements are the major BGP churn contributor for BGP updates. Elmokash et al. [11] introduced a model to predict how BGP churn will grow in the future by considering routing policies, inter-domain topology, and traffic characteristics those changes frequently. The model proved that the number of updates standardised by the size of the topology is constant, and qualitatively related to IPv4 and IPv6. Huston [12] obtained a report on BGP churn from the period 2008 to 2014 and concludes the number of routing updates remains stable for several years, even though the number of prefix in the routing tables are continuously growing. This is explained by the fact that as the Internet grows continuously, the pattern of new connected ASs is repeated through the network topology. It means that the Internet is growing in density rather than in size, resulting in comparatively constant AS path length. The E.A. AiabduIkreem et. al. [13] has expected to modify the path exploration process by maximum reducing the convergence time. To speed up this process authors have used fight or flight response technique. This was carried out by ignoring several parameters when the update message was a withdrawn. Due to this modification, convergence time was reduced by $29 \%$. X. Wang et. al. [14] proposed a technique called Churn Aggregation (CAGG), which tries to decrease path exploration by collecting multiple AS paths in one route, without harming convergence. To aggregate those paths, CAGG nds the Path Locality explored by a highly active prefix, which reduces the total number of exchange updates.

Yu C.W. et al. [15] introduced the concept of alternate/backup/secondary paths along with the main/primary path for which if main route fails then the backup route immediately taken for the further process. But the complexity is more due to managing and storing backup routes. Shivani deshpande et al. [16] introduced a BGP instability detection technique that can be executed by individual routers. The input data for detection of instability is BGP update messages received by routers from its neighbor. From this BGP update messages attributes/features (like AS path length, AS path edit distance) are extracted in every five minutes, this shows the change in topology. The GLR (Generalized Likelihood Ratio test), Boundary position optimization algorithms, Segmentation boundary detection are used to detect the changes. Geo Huston et al. [17] proposed a Path Exploration Damping (PED) mechanism which reduces the number of BGP update messages and also decreases the average time required to restore reachability. They compare PED effect on convergence time with MRAI, Withdrawal Rate Limiting (WRATE), and Route Flap Damping (RFD). Mohammad Yanuar Hariyawan [18] compared different methods like local rerouting, Haskin, 1+1 path protection recovery mechanism, Fast Reroute one to one backs up, and PSL oriented path protection mechanism technique for sooner rerouting after failure. The performance shows that $1+1$ path protection recovery technique has minimum packet loss, but having more cost. Rajvir Gill et al. [19] proposed the FLD-MRAI (Flexible Load Dispersing MRAI) algorithm that disperses the load in the network, which results in reducing the routers load. The authors concentrated on routing policies and their effects on convergence time, number of updates. The FLD-MRAI algorithm works in case of both normal and high loads. When degree of preference (DoP) selects the shortest path, then FLD-MRAI believe this situation as normal load, and when DoP selects the longest path then FLD-MRAI believe this situation as high load.

In a network running the BGP, the end to end reachability information can be temporarily disturbed due to node or link failures and the time required for converging a network lead to service degradation or even interruption, which creates a critical issue for real-time interactive applications. The J. R. Alzate et. al. [20] evaluates the behaviour of ghost flushing and EPIC proposal in Waxman topology networks for reducing the impact of path exploration and MRAI on convergence time of a network. R. N. Devikar et al. [21] surveyed the different issues that are occured at the time of packet transmission. Convergence time is one of the issue in the network that are described in [21]. In [22] author described about the study of convergence time by considering BGP protocol. In this they analyse different techniques and algorithms those are used to improve the convergence time of a network. R. N. Devikar et al. [23] proposed a technique which reduced the convergence time, they also introduced a load balancing algorithm for packet transmission to reduce the congestion in the network. Chen Chen et. al. [24] proposed a mathematical model to compute the BGP convergence time by catching only the important components in BGP convergence process. They further presented a greedy approach that selects ASes for incremental software-defined networking (SDN) deployed with the idea of reducing the BGP convergence time.

The paper tells about the improvement in convergence time and update messages with respect to MRAI timer. Section 2 contains research method, which tells about the Methodology and Algorithm for FMRAI timer using mathematical approach. The result and analysis shown in section 3 contains experimental results. And finally, the last section discuss about conclusion and future scope of the research work. 


\section{RESEARCH METHOD}

To reduce the network convergence time by changing MRAI timer variable dynamically. In the Network, routing update will done after every 30s. Now, if any link failure occur immediately after the routing update of 30s. Then, to get the idea about link failure the node has to wait until next update. During that much time the load on both link failure node increases rapidly. Due to which there may be a chance of packet loss. On making MRAI timer variable changing dynamically. The value of MRAI timer variable can be calculated as early as possible after the link failure or any other change in topology. The value of MRAI will get reduced and will be below 30s which will make the routing updation faster and hence the reduction in convergence time of network.

The following algorithm sets the value of MRAI timer.

$$
\sum P_{i}=\left(p_{1}, p_{2}, p_{3}, p_{4} \ldots \ldots \ldots p_{n}\right)
$$

$\mathrm{P}$ consist of total paths $\mathrm{p}_{1}, \mathrm{p}_{2}, \mathrm{p}_{3}$ $\mathrm{p}_{n}$ those are reaching to the destination D.

Where, $\mathrm{P}_{i}$ is $i^{\text {th }}$ path reaching to destination.

$$
\begin{gathered}
P_{1}=\sum R_{1} \\
\sum R_{1}=\left(r_{1}, r_{2}, r_{3}, r_{4} \ldots \ldots \ldots . r_{n}\right)
\end{gathered}
$$

Every path $P$ consist of large number of routers $\mathrm{R}_{n}$. Suppose path $\mathrm{P}_{1}$ consist of $\mathrm{R}_{1}$ number of routers, Where $\mathrm{R}_{1}$ contains the $\mathrm{r}_{1}, \mathrm{r}_{2}, \mathrm{r}_{3} . . \mathrm{r}_{n}$ router those are available on the path $\mathrm{P}_{1}$.

$R_{1}=$ Total number of routers available on path $P_{1}$

$L\left(P_{i}\right)=\left(l_{1}, l_{2}, l_{3}, l_{4} \ldots \ldots \ldots . . . l_{d}\right)$.

$L\left(P_{i}\right)=$ Total number of links present in path $P_{1}$.

$D\left[L\left(P_{i}\right)\right]=D\left(l_{1}, l_{2}, l_{3}, l_{4} \ldots \ldots \ldots . . l_{d}\right)$

$D\left[L\left(P_{1}\right)\right]=$ Total delay in the links on path $P_{1}$

$\left.W\left(P_{i}\right)\right]=W\left(l_{1}, l_{2}, l_{3}, l_{4} \ldots \ldots \ldots . . . l_{d}\right)$

$W\left(P_{1}\right)=$ Total processing time on path $P_{1}$.

$Q\left[R\left(P_{i}\right)\right]=Q\left(q r_{1}, q r_{2}, q r_{3}, q r_{4} \ldots \ldots \ldots . . q r_{d}\right)$

$Q\left[R\left(P_{1}\right)\right]=$ Total queuing delay at each router on path $P_{1}$

Maximum delay $(T D)=D\left[L\left(P_{i}\right)\right]+W\left(P_{i}\right)+Q\left[R\left(P_{i}\right)\right.$

$M R A I=T D$

if $(T D>=$ Default MRAI $)$

\{

$M R A I=$ Default MRAI;

return MRAI;

\}

else

\{

$M R A I=T D$

return MRAI;

\}

The Convergence time of a network is reduced by introducing the FMRAI (Fickle mean route advertisement interval) timer algorithm shown below. The simulation performed in NS2 simulator by considering the topology shown in figure 1. Topology consist of nodes represent as BGP routers which work as a speaker node for its domain. The 
BGP execution is based on BGP-4 specifications. The network topology is manually created, each node signifies an $\mathrm{AS}$, and all the links are configured in such way that having similar bandwidth and variable delays.
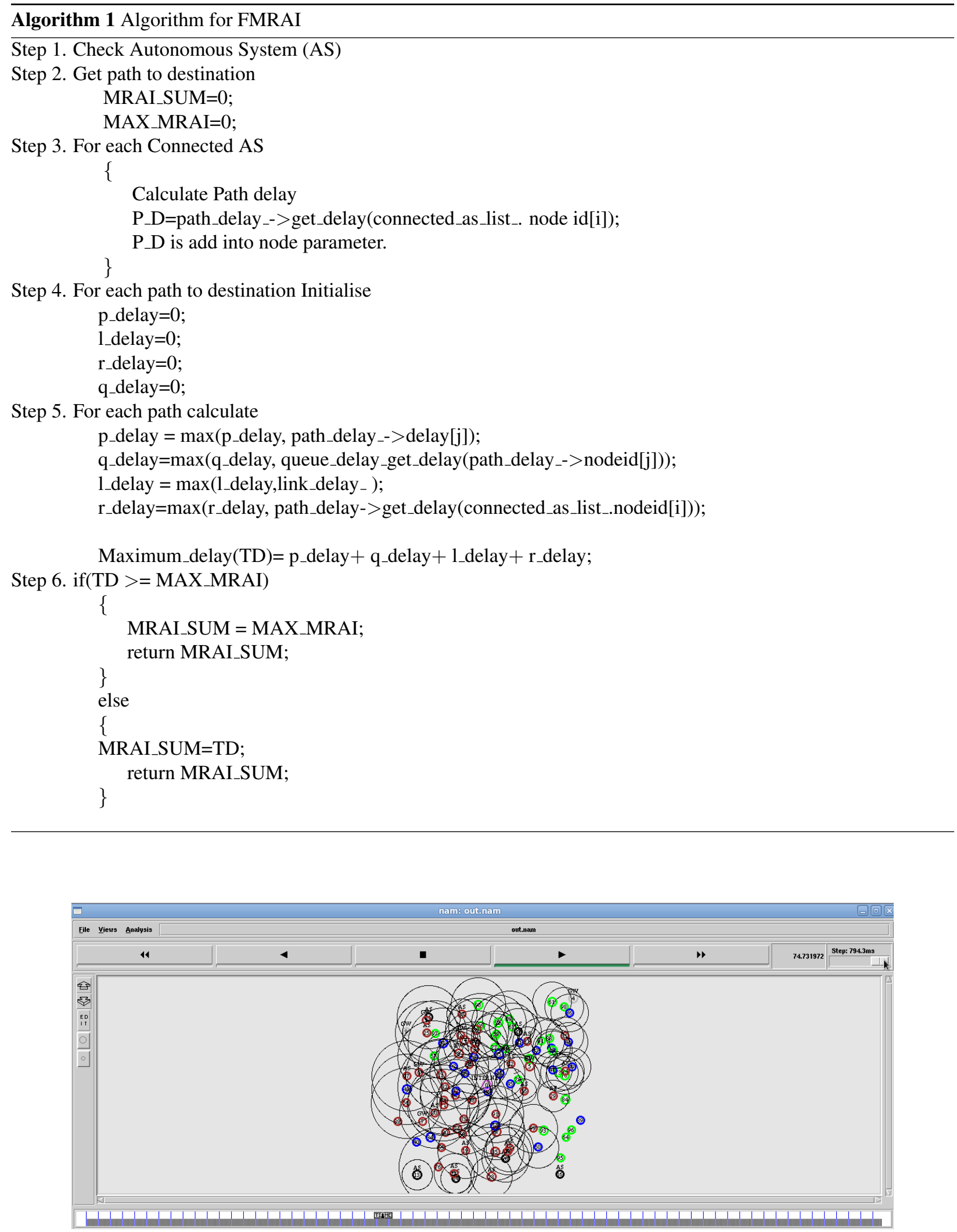

Figure 1. Node Topology 


\section{RESULT AND ANALYSIS}

\subsection{MRAI Vs Convergence Time and Updates}

Convergence time varies with respect to MRAI interval for default as well as FMRAI approach. The convergence time is directly proportional with the MRAI timer which shown in figure 2 . The convergence time increases with increasing MRAI value. Figure 3 shows number of updates decreases with increasing MRAI value. The graph shows two different approaches for increasing MRAI values with respect to number of updates. It shows the FMRAI has more number of updates than default MRAI value. So, to improve the network availability and for fast network convergence it is necessary to keep the value of MRAI timer less. Table 1 shows the network convergence and update activities by considering different MRAI values.

\subsection{Node Vs Packet Delivery Ratio}

The different performance metrics such as packet delivery ratio, end to end delay, routing overhead and throughput are used for analysis of routing protocols [25]. Figure 4 shows packet delivery ratio for varying number of nodes. From the above given results we can say, that default MRAI returns poor result as we start increasing the number of nodes. FMRAI protocols returns best result and thus achieves packet delivery ratio in range of $90 \%$ to $95 \%$. But as we start increasing number of nodes results falls down below 90\%. Table 2 shows the values of packet delivery ratio for different scenario of nodes (Number of Nodes).

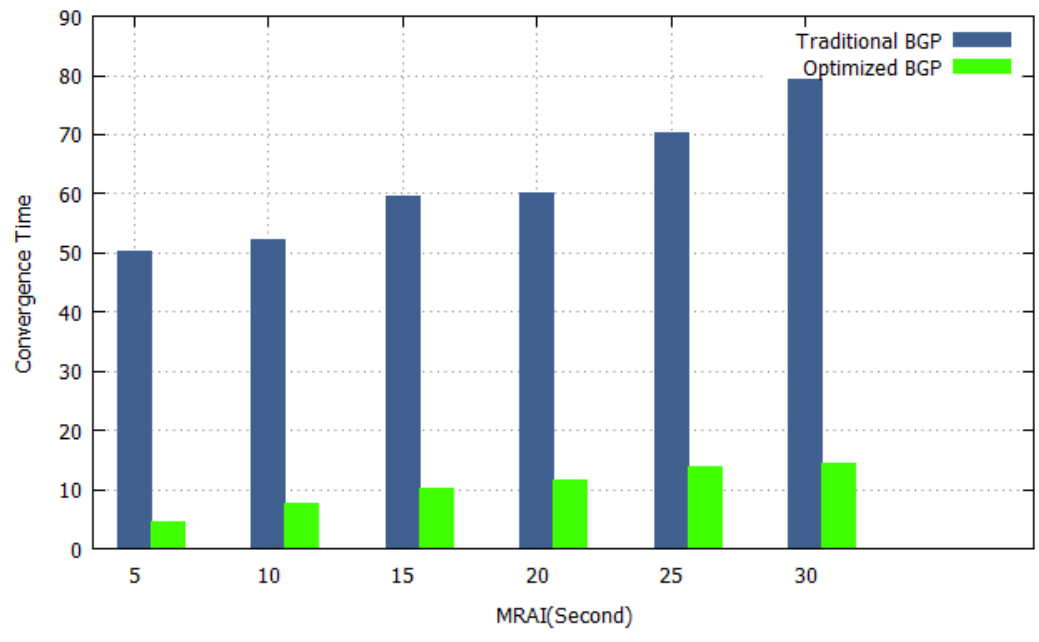

Figure 2. MRAI vs Convergence Time

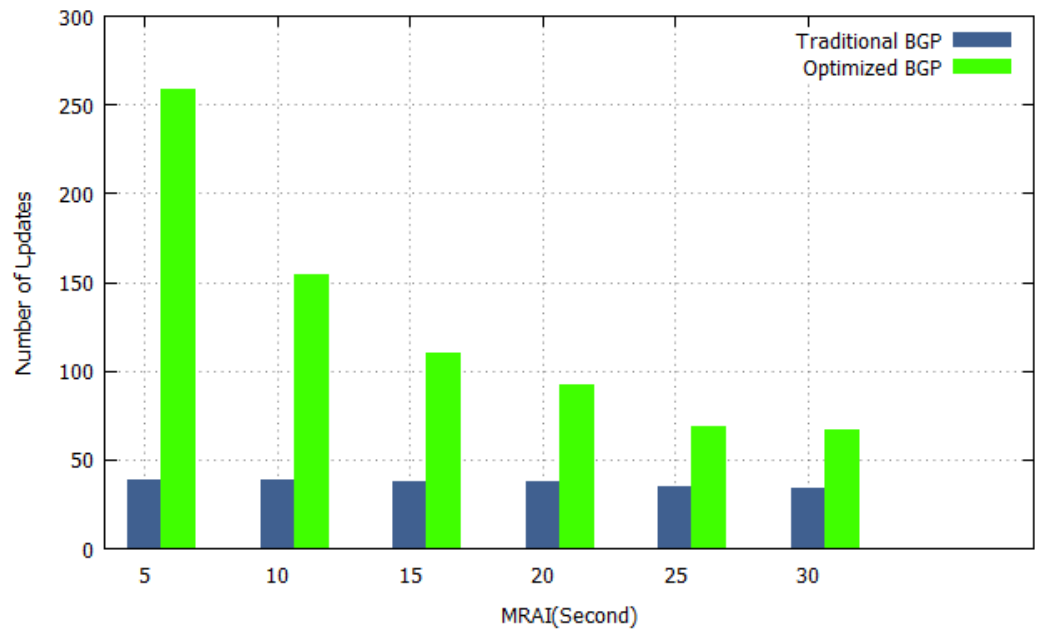

Figure 3. MRAI vs Number of Updates 
Table 1. MRAI vs Convergence time and Number of updates

\begin{tabular}{cccccc}
\hline Sr. No. & MRAI & Traditional BGP & Optimized BGP & Traditional BGP Updates & Optimized BGP Updates \\
\hline 1 & 5 & 50.23 & 4.47644 & 39 & 259 \\
2 & 10 & 52.13 & 7.47462 & 39 & 154 \\
3 & 15 & 59.43 & 10.2025 & 38 & 110 \\
4 & 20 & 60.17 & 11.5735 & 38 & 92 \\
5 & 25 & 70.32 & 13.8668 & 35 & 69 \\
6 & 30 & 79.19 & 14.4961 & 34 & 67 \\
\hline
\end{tabular}

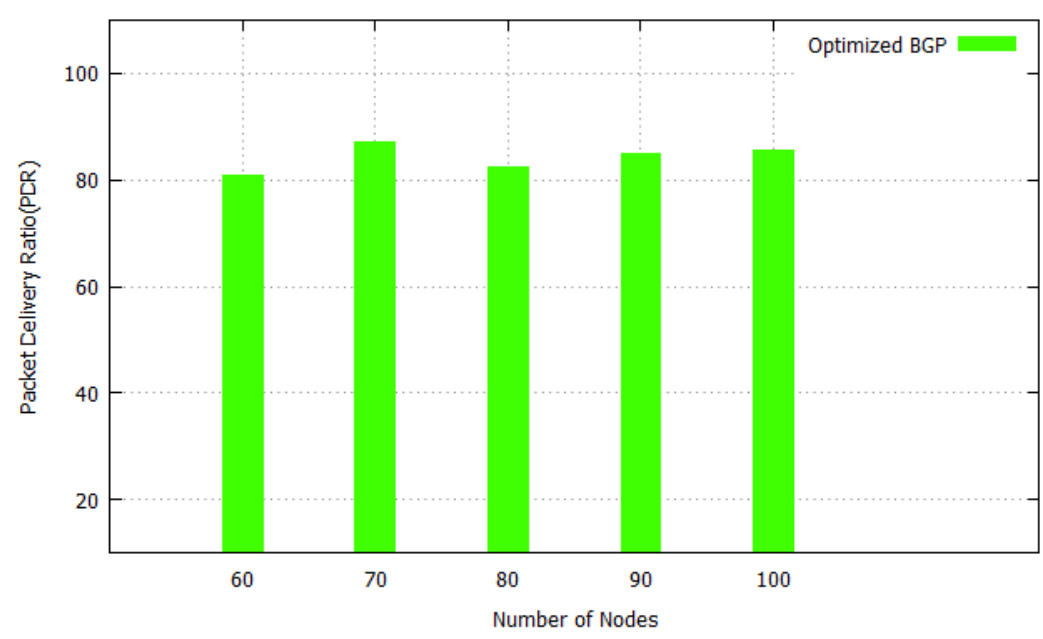

Figure 4. Node vs Packet Delivery Ratio

\subsection{Number of Node Vs Delay}

In this part, we compare the end-to-end delay from the source node to the destination. In Figure 5 the end to end delay varies with number of nodes. The end-to-end delay for network increases much faster than others. When no node is available, then it increases delay of packet transmission. More nodes in network will provide more opportunities to find some suitable node for efficient forwarding of packet by considering potential score of neighbor nodes. With high node density, the transmission delay is dramatically reduced in the network shown in table 2.

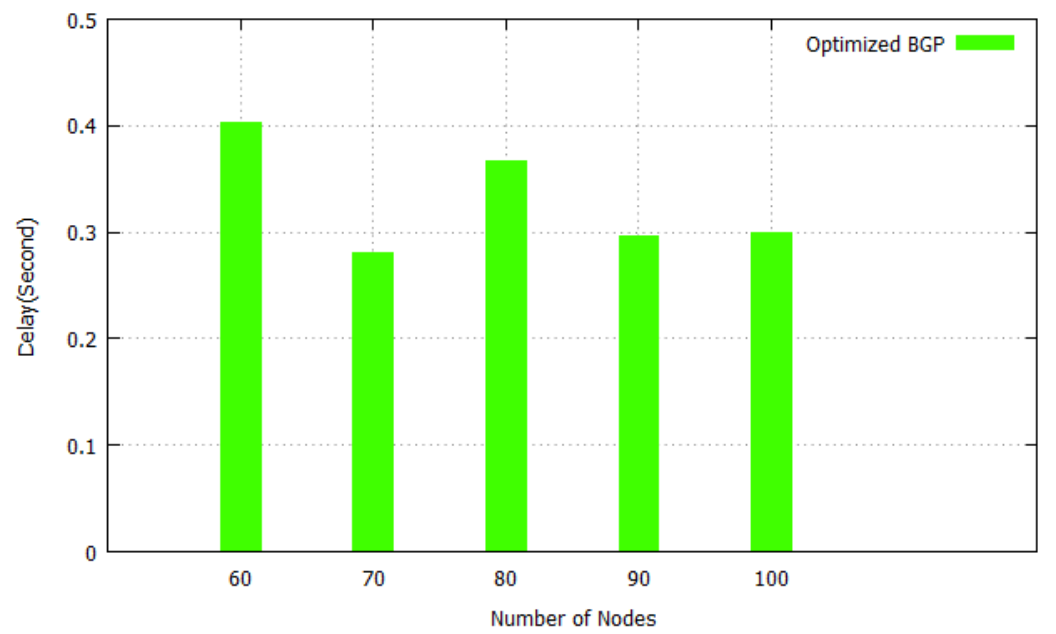

Figure 5. Number of Node vs Delay 


\subsection{Number of nodes vs Number of Updates}

Figure 6 shows number of update messages are varies with respect to number of nodes. As we increase the number of nodes in the network which increases the respective update messages. Tabel 2 shows the comparative results for number of updates in traditional BGP and optimized BGP with respect to number of nodes.

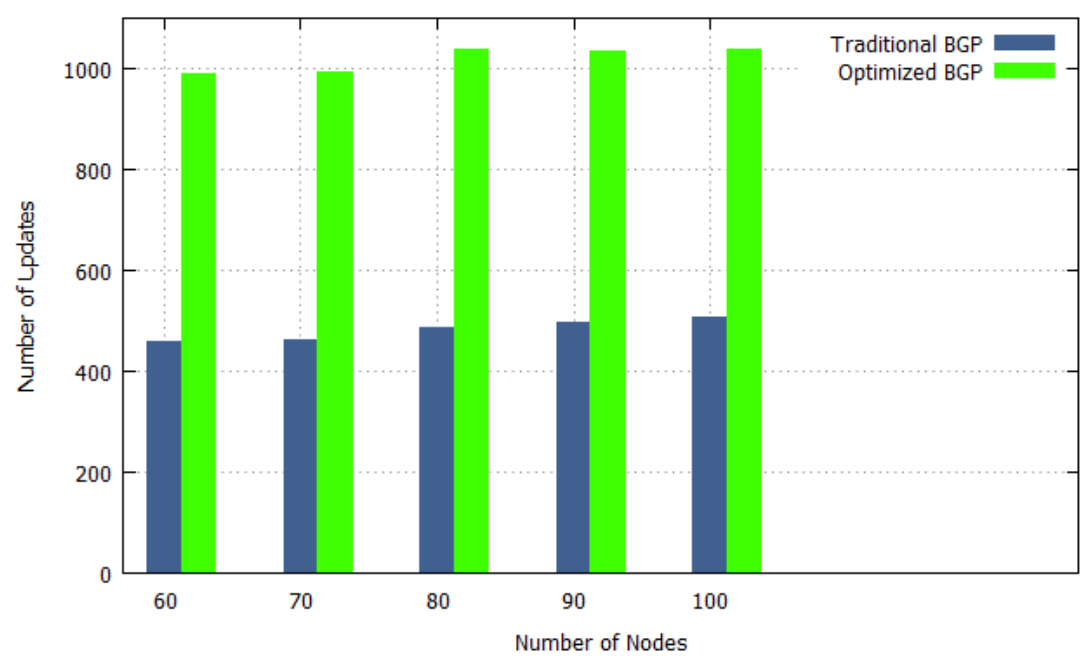

Figure 6. Number of nodes vs Number of Updates

Table 2. Number of nodes vs Number of Updates

\begin{tabular}{cccccc}
\hline Sr. No. & No. of Nodes & Packet Delivery Ratio & Delay & Traditional BGP & Optimized BGP \\
\hline 1 & 60 & 80.7411 & 0.402744 & 457 & 990 \\
2 & 70 & 87.105 & 0.280598 & 462 & 992 \\
3 & 80 & 82.3763 & 0.366363 & 488 & 1039 \\
4 & 90 & 84.7848 & 0.29666 & 498 & 1035 \\
5 & 100 & 85.5714 & 0.300134 & 506 & 1037 \\
\hline
\end{tabular}

\section{CONCLUSION AND FUTURE SCOPE}

The Convergence time of a network is improve by decreasing MRAI timer. But as we decrease it upto zero then in the network only updates are transmitted. The proposed technique Fickle Mean Route Advertisement Interval (FMRAI) use to improve the network convergence time. The FMRAI have lower packet dropping ratio, delay, in comparison with traditional MRAI technique. The proposed algorithm also improves the packet delivery ratio, convergence time, and number of updates compare with tradition technique for different scenarios (Number of Nodes).

In future for improving the convergence time, we will compute Particle Swarm Optimization (PSO) technique, which detects global minimizer to address the problem of fast convergence time.

\section{ACKNOWLEDGEMENT}

I would like to express my deep sense of gratitude towards my research supervisors Dr. V.Chandra prakash and Dr. D. V. Patil for their valuable guidance and encouragement. Also, I would like to thank Mr. Pavan D. Upadhye for his suggestions towards improvement of this paper.

\section{REFERENCES}

[1] McPherson D, Gill V, Walton D, "Border gateway protocol persistent route oscillation condition", RFC3345, August 2002.

[2] Anindya Basu, Chih-Hao Luke Ong, April Rasala, "Route oscillations in I-BGP with route reflection [J]", Computer Communication Review, 2002; 32(4): 235-247. 
[3] https://en.wikipedia.org/wiki/Border Gateway Protocol.

[4] Mahesh Kumar, Shishir Kumar, "Analyzing and Improving Network Availability in a Interdomain Routing”, in Applied Power Electronics Conference and Exposition, 2009. APEC 2009. Twenty-Fourth Annual IEEE, 2009; 128-131.

[5] T. Grifn, B. Shepherd, and G. Wilfong, "The stable paths problem and interdomain routing", IEEE/ACM Transactions on Networking, 2002; 10: 232243.

[6] L. Cittadini, M. Rimondini, M. Corea, and G. Di Battista, "On the feasibility of static analysis for BGP convergence", in IFIP/IEEE International Symposium on Integrated Network Management- IM 09, 2009; 521528.

[7] A. Fabrikant, U. Syed, and J. Rexford, "Theres something about MRAI: Timing diversity can exponentially worsen BGP convergence”, in INFOCOM11, 2011; 29752983.

[8] A. Elmokash, A. Kvalbein, and C. Dovrolis, "On the scalability of BGP: The roles of topology growth and update rate-limiting", in Proceedings of the 2008 ACM CoNEXT Conference, (New York, NY, USA), pp. 8:18:12, 2008.

[9] V. Schrieck, P. Francois, C. Pelsser, and O. Bonaventure, "Preventing the unnecessary propagation of BGP withdraws", in Proceedings of IFIP Networking Springer Verlag, 2009; 495508.

[10] A. Elmokash, A. Kvalbein, and C. Dovrolis, "BGP churn evolution: A perspective from the core", IEEE/ACM Transactions on Networking, 2012; 20: 571584.

[11] A. Elmokash and A. Dhamdhere, "Revisiting BGP churn growth", SIGCOMM Comput. Commun. Rev., 2013; 44: 512

[12] G. Huston, “The Churn Report”, https://labs.apnic.net/?p=457, 2014.

[13] E.A. AiabduIkreem, H. S. Al-Raweshidy, and M. F. Abbod, "Using a Fight-or-Flight Mechanism to Reduce BGP Convergence Time", International Conference on Communications and Networking (ComNet), 19-22 Mar 2014.

[14] X. Wang, O. Bonaventure, and P. Zhu, "Stabilizing BGP routing without harming convergence", in IEEE Conference on Computer Communications Workshops (INFOCOM WKSHPS), 2011; 840845.

[15] Yu K.-M., Yu C.W.and Yan S.-F. , "An ad hoc routing protocol with multiple backup routes”, Wireless Personal Communications, 2014; 57(4); 533-551.

[16] Shivani Deshpande, Marina Thottan, TinKamHo, and Biplab Sikdar, "An Online Mechanism for BGP Instability Detection and Analysis”, IEEE Transactions on Computers, 2009: 58(11); 1470-1484.

[17] Geo Huston, Mattia Rossi, and Grenville Armitage, "A Technique for Reducing BGP Update Announcements through Path Exploration Damping”, IEEE Journal on Selected Areas in Communications, 2010: 28(8); 12711286.

[18] Mohammad Yanuar Hariyawan, "Comparison Analysis of Recovery Mechanism at MPLS Network", International Journal of Electrical and Computer Engineering(IJECE), 2011:1(2); 151-160.

[19] Rajvir Gill, Ravinder Paul, and Ljiljana Trajkovic, "Effect of MRAI Timers and Routing Policies on BGP Convergence Times", In proc. IPCCC, IEEE 31st International Conference, 1-3 Dec 2012.

[20] Jackson Reina Alzate; Roberto Carlos Hincapi Reyes, "Evaluation of improvement proposals for Border Gateway Protocol (BGP)", IEEE Colombian Communications Conference (COLCOM),16-18 May 2012: 1-6.

[21] Rohit Nilkanth Devikar, Dipak V. Patil, and V. Chandra Prakash, "Issues in Routing Mechanism for Packet Forwarding: A Survey”, International Journal of Electrical and Computer Engineering (IJECE), 2016: 6(1); 421430.

[22] Rohit Nilkanth Devikar, Dipak V. Patil, and V. Chandra Prakash, "Study of BGP Convergence Time", International Journal of Electrical and Computer Engineering (IJECE), 2016: 6(1); 413-420.

[23] Rohit N. Devikar, D. V. Patil and V. Chandra prakash, "A Mathematical Approach to Improve the Network Performance", Indian Journal of Science and Technology,2016: 9(2).

[24] Chen Chen, Bo Li , Dong Lin, and Baochun Li, "Software-Dened Inter-Domain Routing Revisited", IEEE International Conference on Communications (ICC), 22-27 May 2016.

[25] Anton Pavlovich Teykhrib, "Data transmission in Hybrid Distributed Environment", International Journal of Electrical and Computer Engineering (IJECE), 2016: 6(6); 2989-2993. 


\section{BIOGRAPHY OF AUTHORS}
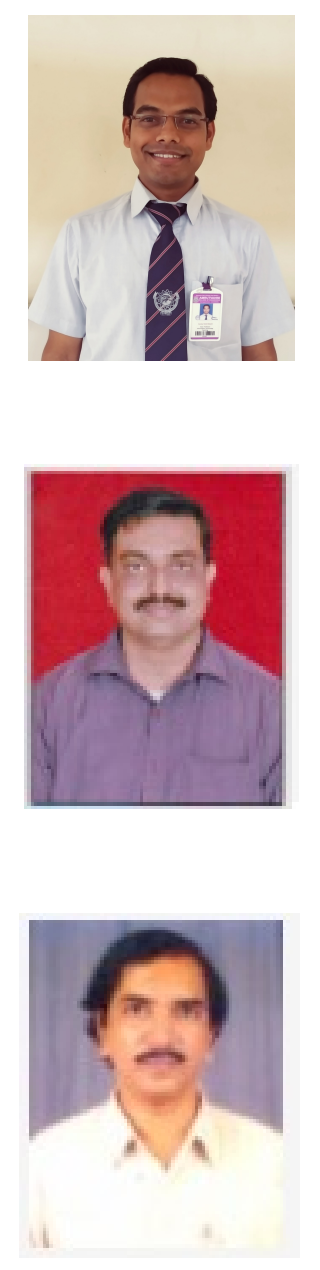

Rohit Devikar obtained Bachelor of Engineering degree in Computer Engineering from Savitribai Phule Pune University (M.S.), India, in 2010. Also he received Master of Technology degree in Computer Engineering from Dr. BATU, Lonere, (M.S.), India in 2012. Currently he is a PhD Research Scholar in Computer Science and Engineering in K. L. University, Vijayawada. He is working as Assistant Professor in Amrutvahini College of Engineering, Sangamner, (M.S.), India. His major area of interest is Congestion Control, Availability of network, Load Balancing, Convergence speed etc.

E-mail: rohit.devikar89@gmail.com (Corresponding author)

D. V. Patil received the $\mathrm{PhD}$ degree in Computer Science and Engineering from the S.G.G.S. institute of Engineering and Technology, S.R.T.M.U., Nanded, Sept. 2013. He is presently a Professor and Head of Computer Engineering department in R. H. Sapat College of Engineering, (M.S.), India . His research interests include Data center, Soft computing, Distributed processing, and Data Mining.

E-mail: dipakvpatil17@gmail.com

V. Chandra Prakash received the $\mathrm{PhD}$ degree in Computer Science and Engineering from Acharya Nagarjuna University in 2011. He is presently working as Professor of Computer Science and Engineering department in K. L. University (A.P.), India. His research interests include Software Engineering, Software Testing, Soft Computing, Artificial Intelligence and Data Mining.

E-mail: vchandrap@kluniversity.in 